\title{
Research on Quality and Safety Problems and Solutions in Grain Collection and Storage and Logistics Transportation
}

\author{
Guigen Pan
}

Jiangxi Vocational Technical College of lndustry \& Trade, Jiangxi, Nanchang,330038

3107000933@qq.com

\begin{abstract}
As a large agricultural country, China often needs to pay attention to the problem of quality and safety in the process of grain storage and logistics transportation. At present, China's food safety mainly aims at these two aspects to find solutions. This paper mainly focuses on the quality and safety problems and Countermeasures in grain collection and storage and logistics transportation. This paper mainly starts from three aspects of grain, including purchase, storage and transportation, observes the existing quality and safety problems, analyzes them, and puts forward corresponding countermeasures and methods.
\end{abstract}

Keywords: grain collection and storage, Logistics transportation, Quality and safety issues, countermeasure

\section{粮食收储及物流运输环节存在的质量安全问题和解决 办法研究}

潘桂根

江西工业贸易职业技术学院 江西 南昌 330038

3107000933@qq.com

摘要:

我国作为农业大国, 在对粮食进行储备以及物流运输过程中往往需要重视质量安全的问题, 目前我国食品安全 主要针对这两个方面来对寻找解决措施。本文主要在于探究粮食收储及物流运输环节存在的质量安全问题与 对策。本文主要从粮食的三个方面出发, 其中就包括收购、储备、运输, 观察其中存在的质量安全问题, 并对 其进行分析提出相应的对策与方法。

关键词: 粮食收储; 物流运输; 质量安全问题; 对策

\section{1.粮食收购过程中存在的质量安全问题}

目前, 我国社会不断进步, 人们的生活水平不断 提高, 人们越来越重视粮食安全问题, 现在不需要每 家每户都去种植粮食, 目前, 人们所需的粮食大多是 像其他人或者是地方采购的, 大量的粮食在收储以及 运输的过程中, 涉及到多个环节, 在这过程中, 粮食 会受诸多因素的影响, 导致粮食质量安全存在隐患, 因此, 有关单位应当对这些环节加大监督的力度, 控
制危险因素, 从而保证粮食的质量安全。为了避免粮 食出现质量安全问题, 我们应当积极寻找科学的解决 办法, 制定相应的标准以及制度来减少影响粮食质量 安全因素的影响。在粮食收储以及物流的过程中, 受 条件限制, 粮食堆放的环境不够干燥, 存储技术落后 等等, 都是粮食质量安全问题产生的原因。为保障粮 食质量安全, 本文将针对粮食收储以及物流的过程中 存在的问题, 一一展开分析, 有针对性的提出解决措 施, 为实际应用提供理论依据。 


\section{1 粮食干燥条件限制}

对于粮食收成的长期保存工作来说，前提条件就 必须是经过干燥处理。目前从国家的粮食保存情况来 看, 可以发现以下几点问题: 一是由于场地与设备的 限制使得粮食整体的质量与含水量常常不能达标, 从 而引发一系列的问题, 其中就包括后期霉变, 出现一 部分污染等等; 二是对于负责对粮食进行干燥工作的 农民来说, 一般情况下都会使用晾晒干燥的方法去处 理, 这类干燥方法往往会使得粮食的安全性降低, 并 且有概率会出现受有害物质影响的情况, 比如多环芳 烃等等, 虽然也具有干燥效果, 但是容易造成后期的 生产加工危害。

\section{2 对粮食品质的检验能力较差}

国家现有法律条例中已经针对粮食中所含的农 药残留、真菌毒素以及重金属等的限量标准做出了具 体说明, 所以对于具体收购过程中, 工作人员往往会 针对粮食的质量进行检验, 只有最终结果能够符合国 家相关标准才可以开始下一步的工作行动, 一般情况 下, 往往根据具体的实际情况去对粮食质量进行严格 检查的相关单位并不多, 出现这种现象的主要原因, 主要有两点: 一是检验方法的不完善, 达不到预期标 准; 二是大部分员工在收购方面素质较低, 难以完成 任务或者达到标准。由于这些原因的出现, 往往会伴 随出现问题也会更加严峻, 其中就包括粮食重金属超 标以及农药残余等问题, 倘若这些粮食流入市场交易, 将造成不可估量的影响。

\section{2.粮食存储过程中存在的质量安全问题}

粮食存储过程中存在的质量安全问题主要包括 以下这三种: 不能有效利用存储技术; 违规使用化学 药剂以及很多仓库中存在交叉污染。

\section{1 不能有效利用存储技术}

在粮食存储的过程中, 不同的存储方式、不同的 存储环境、温度, 湿度的差异都会对粮食的质量产生 一定的影响, 因此, 在进行粮食存储时, 应当根据实 际的存储环境, 选用恰当的存储技术, 保证粮食的治 疗。

对于粮食的储备来说, 实际的存储过程往往会有 所不同。粮食在存储期间在一定空间内会和其他的生 物与非生物形成新的生态系统, 假若缺失部分元素, 比如气体、杂质、害虫等等, 都会使得系统出现问题 而导致粮食出现安全问题。面对这样的安全问题, 相 关部门和政府在重视粮食储备问题的同时, 也出台了 关于粮食储存的具体办法与规范标准, 如果工作人员 不能达标, 就无法和前者结合发展。因此粮食的整体 质量将会下降, 从而出现安全隐患。

\section{2 违规使用化学药剂}

一般情况下，在存储粮食的过程中，为了消灭一 些有害物质或者有害生物会适当使用一些化学药剂, 但化学药剂的使用必须严格控制使用量, 用量过少难 以实现消灭的效果, 用量过多会污染粮食, 造成药物 残留, 影响到粮食的质量安全。就拿敌敌畏来说, 该 药物本身具有良好的触杀熏蒸效果，但在实际应用的 过程中, 该药物在存储环境中不易扩散, 造成药物残 留在食物中, 因此, 在选择何种药剂时, 需要全面考 虑, 兼顾方方面面, 避免一些不必要的问题 ${ }^{[4]}$ 。

\section{3 仓库中存在交叉污染}

大量粮食在收购过程中, 在没有正式运输到粮食 仓库之前,一些单位就会选择性去租借商业仓库去临 时性储存, 在选择的过程中往往不会去针对性对商业 仓库进行篎选，没有考虑到是否储存过其他物体等等， 这些没有重视的因素就成为了影响粮食质量安全的 重要因素。对于这类问题的出现, 现有出行的粮食存 储管理办法就已经有了相应的解决说明, 其中明确的 指出仓库周围是否放置或者存在易燃易爆危险物、有 污染的化工企业等等都是十分影响粮食安全问题的 隐患, 曾存放过有毒物体以及活物的仓库, 只有在进 行彻底消毒工作以后才可以存放粮食, 否则就会出现 严重的粮食储备污染问题。

\section{3.粮食运输过程中存在的质量安全问题}

\section{1 对粮食运输质量安全问题的关注程度不够 高}

我国在农业发展领域是农业大国，但是本身的粮 食物流发展仍旧是起步阶段, 面对实际的运输情况, 往往就会存在成本高、效率低以及品种混乱等问题, 这些问题的出现对于我国目前的粮食运输发展有着 重要影响。目前绝大多数的运输单位在对粮食进行运 输的过程中往往只会去重视粮食在运输过程中减少 粮食损耗，没有发现运输中粮食的安全与质量问题， 很多隐患的出现使得最近几年的粮食运输出现许多 粮食质量问题。因此对于粮食运输的关注度不够, 导 致粮食运输难以发展。

\section{2 长距离运输方案中依然存在缺陷}

针对长距离运输方案, 本文主要以东北地区到南 方销售区粮食运输为例, 对整体的运输进行分析, 本 次运输总时长是在 20 30d, 由于北方与南方的各类 情况不同, 导致粮食整体在运输过程中会受到温度差、 湿度差的影响。面对这种情况, 粮食往往基本都不能 食用, 对于南方销售区为中心的消费者群体来说会对 生活造成影响。因此, 必须重视长距离运输过程存在 的各个因素，然后计划制定相应的方案，才可以最大 程度上减少粮食质量的隐患。 


\section{4.粮食收储及物流运输环节存在的质量安全 问题的解决办法}

通过对已有的资料进行分析，就粮食收储及物流 运输环节存在的质量安全问题, 可以从一下这几个方 面入手: 在原有基础上强化农业标准化生产的发展; 注重对绿色储粮技术的应用; 对传统的散粮运输方式 进行改善以及构建完善的粮食安全追溯体系。

\section{1 在原有基础上强化农业标准化生产的发展}

农业标准化生产是对粮食生产的三个阶段进行 经营管理，根据农业标准化生产的有关规定，采取标 准化生产技术, 使得粮食生产的规模化、规范化、产 业化等将能更有效地达到预期目标。我国农业标准化 的生产往往都需要通过 HACCP 来进行发展。所谓 HACCP 就是对具体危害以及关键控制点的分析, 对 于标准化生产来说这一体系的主要内容几乎都需要 应用, 只有这样才能够去保证粮食质量, 同时也能够 快速的把握住粮食储存、运输中所存在的各种影响因 素, 在此基础上采取相应的措施, 真正意义上对粮食 质量安全进行保护。结合实际情况, 相关单位必须把 HACCP 和 GMP 融合发展起来, 从多个方面去进行 标准化控制, 其中就包括土壤、种子、农药、运输等 等, 因此就必须建立健全我国独有的专业标准化生产 体系，才能保证国家粮食质量的标准。

\section{2 注重对绿色储粮技术的应用}

我国在针对粮食存储方面已经开始使用控温储 粮技术、充氮气调储粮技术等, 这些技术的投入带来 了可观的效果, 因此在未来的粮食质量安全发展过程 中, 相关单位就应该去重视这些技术重要性, 针对性 的对工作人员对这些技术进行引导与培训, 保障在工 作过程中能够有效发挥优秀技术的作用并在粮食质 量安全上取得效果。就那控温储粮技术来说, 该技术 主要是通过相应的手段来实现对温度进行控制, 其中 就包括过压盖、隔热保冷、气囊控温、空调控温等, 由于粮食始终保持在低温环境中, 整体变化缓慢, 有 效的防止粮食霉变。充氮气调储粮技术的运用, 主要 的特点在于无污染, 不会对工作人员的生活造成影响, 当然它还具有其他的优势, 能够有效的抑制住霉菌的 产生, 但是本身需要保持标准较高的密闭性作为基础。

\section{3 对传统的散粮运输方式进行改善}

目前我国传统散粮的运输方式仍旧存在很多问 题, 因此往往需要针对以下几点进行改善。首先是必 须建立粮食运输专业的合作组织, 由于目前国家运输 散粮中的主要问题在于次数多、单次运输量少、以及 没有形成规模化经营, 所以就需要形成多个运输粮食 企业合作发展, 在国家的标准下形成粮食运输专业组 织, 加快我国散粮的运输效率与质量。其次, 必须建 立健全一体化的运输模式, 该模式的实行能够有效的
保证散粮运输的质量、数量以及效益等等, 该模式的 一体化主要体现在运输设备、运输方式等多个方面的 一体化, 重点在于以散粮集装箱多式联运为主、粮食 运输车辆为辅的综合运输一体化模式是该模式最为 重要的基础保障。

\section{4 构建完善的粮食安全追溯体系}

粮食安全追溯体系的出现能够有效保证国家粮 食在实际的运输过程中，找到关键性的问题并解决。 目前国家粮食品种繁多，假如从粮食品种的分类出发 去建立安全追溯系统, 不仅仅会增加大量的人工成本 和物质成本, 还不能够发挥出较好的作用与效果。因 此, 有关单位以及政府有关部门就应该在建立粮食安 全追溯体系的过程中去学习和运用 HACCP 的思想, 找到关键点, 例如对于粮食收储企业来说, 往往可以 从粮食入库时就开始管理, 有效的为餐桌可追溯发下 基础。

\section{5 结论}

总的来说, 我国的人口众多, 对于我国来说粮食 的收储与运输是十分关键的, 不仅仅需要去保证粮食 质量安全, 也需要有关部门及单位去加强管理和把控, 利用先进的技术去加强农业的标准化生产与绿色储 粮, 建立健全粮食一体化模式, 使我国粮食质量安全 得以保障。

\section{REFERENCES}

[1] Cao Jun, Liu Xin, Chen Wenruo, Dai bingye, Jiang Weixin, Dong Wen, Chen Yinji. Research progress on physiological metabolism and quality change mechanism of corn during storage [J]. Food industry science and technology, 2016,37 (3): 379-383

[2] Wang Xin, Cui Lijun, Pan Zhi, Tang Xinyu, Yang Gang, Duan Lei, Ma Baoying, Yang Shuangmei, Chen Yu. On site drying of high moisture corn with high temperature and low humidity air [J]. Grain and oil storage technology communication, 2013,29 (6): 14-16

[3] Liang Lanlan, Zhao Zhimin, Wu Junhui, Wu qiuting, Chen Jiadong, Ning Zhengxiang. Effect of rice aging time on quality characteristics of rice flour products [J]. Journal of South China University of Technology: Natural Science Edition, 2010 (4): 6570

[4] Chen Xiaoping, Wang Li, sun Xiaoyong, Chen Xiangyang, Li Yuan, Wang Sulan, Lei Jianfang, Ma Shulin, Xue Guang County, Yin Xiaocheng, Zhang lailin, Liu Hui. Mechanical ventilation and precipitation test of high moisture corn aboveground cage [J]. Grain storage, 2007,36 (3): 36-39 
[5] Shi Jianfang, Zhai Xiaona, Wang Xiaomeng, Shao Guang, Zhao Huining, Xie Qizhen. Discussion on postpartum grain quality and quality assurance transportation technology in Northeast China [J]. Grain, oil and food science and technology, 2021,29 (2): $200-210$

[6] Zhou Tianzhi, Liu Chucai, Li Weimin, Liu Ailan, Wang Qingming, Xia Hongdian, Liu Guihua, Zhang Yong. Study on quality changes of Indica Rice with different water content under different storage temperatures [J]. Grain storage, 2005,34 (3): 30-35

[7] Gu Guangdong, Cao Lei, Liu Chao, Zhu Changbao, Zhou Jian, pan Yuedong, Wang Yi, Cao Shengnan, Wu Xianshan, Song Yu, Tao Shu, Hong Ying, Duan Yimeng, Zhang Weihua. Analysis of factors affecting grain on-site drying effect [J]. Grain and oil storage technology communication, 2020,0 (1): $22-27$

[8] Xiao Jianwen, Zhang lailin, Jin Wen, Lian Guirong, Li Yanfeng, Fu Gang, Liu Jinming. Study on the effect of nitrogen charging on maize quality [J]. Journal of Henan University of Technology: Natural Science Edition, 2010,31 (4): 57-60

[9] Shi Jianfang, Zhai Xiaona, Wang Xiaomeng, Shao Guang, Zhao Huining, Xie Qizhen. Discussion on postpartum grain quality and quality assurance transportation technology in Northeast China [J]. Grain, oil and food science and technology, 2021,29 (2): 200-210

[10] Zhang lailin, Qu chenling, Li Jianfeng, Yuan Hongbin, LAN Hongjun, Liu Chang, Wang Jilong, $\mathrm{Fu}$ along. Study on new grain storage technology of "temperature control and water conservation" for high moisture corn I. simulation test of shallow ventilation and precipitation of grain pile [J]. Journal of Henan University of Technology: Natural Science Edition, 2019,40 (1): 94-97

[11] Xu Yong'an, he peihuan, Li Fujun, Cao Yang, Zheng Dan, Chen Xin, an yinghan. Discussion on quality control in the process of grain collection, storage and circulation [J]. Grain storage, 2017,46 (3): 49-52

[12] Yang Guofeng, Yang Huiping, Yang Jin, Wang Ruolan, Wang Dianxuan, LAN Shengbin, he Xuechao, Wan Qing. Overview of grain quality control and grain circulation technology in Canada [J]. Grain storage, 2001,30 (4): 42-48

[13] Zhang Lifeng, LV Zhiyuan, Li Jianrong. Study on the optimization of logistics operation mode of fresh agricultural products in Liaoning Province $[\mathrm{J}]$. Journal of Bohai University: Philosophy and Social Sciences Edition, 2016,38 (1): 50-54
[14] Zhang lailin, Qu chenling, Li Jianfeng, Yuan Hongbin, LAN Hongjun, Liu Chang, Wang Jilong, $\mathrm{Fu}$ along. Study on new grain storage technology of "temperature control and water conservation" for high moisture corn I. simulation test of shallow ventilation and precipitation of grain pile $[\mathrm{J}]$. Journal of Henan University of Technology: Natural Science Edition, 2019,40 (1): 94-97

[15] Yang Lu, Zhao Jiangtao, Tan Huize, Liu Songbai, Dong Ying, Feng Jianwen, Chen Li, Chen Ling, Huang Haitao, Wang Xiaoqiong. Advances in aging mechanism and quality determination of stored corn [J]. Feed research, 2016,0 (24): 49-53

[16] Xiu Lin, Chang Pengfei, Zheng Mingzhu, Cai Dan, Zhang Dali, Liu Jingsheng. Effects of simulated summer storage conditions on starch and amylase activities in corn grains [J]. Grain and feed industry, 2016,0 (11): 5-8 